\title{
Passivity-Based Observer Design for a Class of Lagrangian Systems with Perfect Unilateral Constraints
}

\author{
Aneel Tanwani, Bernard Brogliato and Christophe Prieur
}

\begin{abstract}
This paper addresses the problem of estimating the velocity variables, using the position measurement as output, in nonlinear Lagrangian dynamical systems with perfect unilateral constraints. The dynamics of such systems are formulated as a measure differential inclusion (MDI) at velocity level which naturally encodes the relations for prescribing the post-impact velocity. Under the assumption that the velocity of the system is uniformly bounded, an observer is designed which is also a measure differential inclusion. It is proved that there exists a unique solution to the proposed observer and that solution converges asymptotically to the actual velocity.
\end{abstract}

\section{INTRODUCTION}

In this paper, we consider mechanical systems with unilateral constraints (without friction) on the position of a moving point, whose position and velocity we choose to denote by $q$ and $\dot{q}$, respectively. The unconstrained motion of the particle satisfies the equation

$$
\ddot{q}=F(t, q, \dot{q}),
$$

and the position $q$ is constrained by:

$$
h_{i}(q) \geq 0, \quad i \in\{1, \ldots, m\}
$$

where $F: \mathbb{R} \times \mathbb{R}^{n} \times \mathbb{R}^{n} \rightarrow \mathbb{R}^{n}$ denotes a vector field, and $h_{i}: \mathbb{R}^{n} \rightarrow \mathbb{R}$ represent the constraints imposed on a moving particle. Mechanical systems with impacts, such as robots and colliding rigid bodies could be seen as systems with unilateral constraints. It is seen that the nonsmooth behavior, or discontinuity in state trajectory, appears in such systems when any of the constraint is active, that is, $h_{i}(q)=0$. This is because the velocity must change its direction instantaneously to keep the moving point inside the admissible set. In general, it is seen that the trajectories of such systems are algebraically constrained, they exhibit continuous (due to flow within the interior of the set) as well as discrete dynamics (due to impacts with the boundary of the set), and hence form an important class of nonsmooth systems. There are several modeling frameworks for such nonsmooth systems; one such modeling framework, which is used to model the motion of state-constrained trajectories is the so-called sweeping process [11]. The term so-coined because they represent the motion of a point inside a closed set. As the the set moves, the point is swept across by the moving set. If for such processes, the constraint set is

A. Tanwani and B. Brogliato are with Team BipOp at Institut National de Recherche en Informatique et Automatique (INRIA), Rhône-Alpes, France. Email:\{aneel.tanwani, bernard.brogliato\}einria.fr

C. Prieur is with Department of Automatic Control, Gipsa-lab, 961 rue de la Houille Blanche, BP 46, 38402 Grenoble Cedex, France. Email: christophe.prieur@gipsa-lab.grenoble-inp.fr parameterized by time only, then we call it the first-order sweeping process. However, for system (1), we first define an admissible set for velocity $\dot{q}(\cdot)$ which is parameterized by the state $q(\cdot)$, and this formulation leads to a second-order sweeping process.

This paper is concerned with the design of observers for estimating the velocity $\dot{q}(\cdot)$ using the position $q(\cdot)$ as the output, while using the sweeping process formulation to describe the dynamics of the system and the observer. The construction of observers, or state estimators, is a classical problem in the design of control systems and has found many useful applications such as output feedback control, and fault diagnosis. For smooth systems, there are several standard techniques such as Kalman filter, Luenberger observer, or high-gain approach. Such techniques have also been applied to design observers for certain classes of smooth and unconstrained Lagrangian systems with application to output feedback control, see for example, [3], [4], [16], [27]. A common element of these designs is to assume that the velocity $\dot{q}(\cdot)$ is uniformly bounded (in time) which is primarily because $F(t, q, \cdot)$ is quadratic in general.

Lately, the researchers have started looking at the stateestimation problem in nonsmooth systems. In this regard, we mention the recent work on observer design of switched systems with ordinary differential equations [21], [22], [24], switched differential-algebraic equations [25], [26], certain classes of differential inclusions [6], [17], [23], complementarity systems [10], and the references therein for more details. Classical approaches for observer design are based on constructing an auxiliary dynamical system driven by the error between the measured output and the estimated output, where it is shown that the resulting dynamics of the state estimation error converge to the origin. However, for nonsmooth systems subjected to impacts, such schemes are not easily implementable since the impacts, or discrete dynamics, are not influenced by error injection and hence destroy the integration effect.

For nonsmooth Lagrangian systems with impacts, the problem of state estimation has been considered in [12] under certain restrictive assumptions, and state estimation with tracking control in [9] for motions restricted within a convex polyhedral domain. This article, however, deals with a more general class of nonsmooth Lagrangian dynamics, that allow more general domains, using the formalism of differential inclusions. The approach adopted is closely related to the observer design presented in [6]. The authors in [6] work with differential inclusions that represent the first-order sweeping process and the resulting trajectories are 
absolutely continuous. Since the constraint set is a function of time, this information is passed to the state estimator. The state estimator replicates the system dynamics, and hence is a sweeping process of the first-order. The convexity of the constraint set then generates the passivity relation between the error dynamics and the output estimation error, which is the key component in proving the error convergence.

In this paper, however, the system under consideration is a second-order sweeping process. This way, the statetrajectories of the system are allowed to be of locally bounded variation (BV), and hence discontinuous, which introduces the major difference. Since locally BV functions may admit an infinite number of discontinuities in finite time, the Zeno phenomenon is not excluded in our setup. The proposed observer only describes the dynamics for the velocity estimate (and not the position) in the form of a differential inclusion driven by the measured output (position). It is proved that, for each output, there exists a unique solution to such differential inclusions, and that this solution converges to the actual velocity of the system asymptotically.

\section{PRELIMINARIES}

In this section, we collect some basic definitions and notations that will be used later on. The total variation of a function $f:[a, b] \rightarrow \mathbb{R}^{n}$ is defined as $\operatorname{var}_{f}(\tau)=$ $\sup \sum_{i=1}^{k}\left|f\left(t_{i}\right)-f\left(t_{i-1}\right)\right|, \tau \in[a, b]$, where the supremum is taken over all integers $k \geq 1$, and all possible choices of the sequence $\left\{t_{i}\right\}$ such that $a=t_{0}<t_{1}<\cdots<t_{k}=\tau$. The function $f(\cdot)$ is said to be of bounded variation (BV) on $[a, b]$ if $\operatorname{var}_{f}(b)<\infty$. If it is right continuous with bounded variation we denote it with $r c b v$. It is locally $r c b v$ if this holds for any bounded interval $[a, b] \subset \mathbb{R}$. If $f(\cdot)$ is $\mathrm{BV}$ on $[a, b]$ then it has countable number of jump discontinuities. Moreover, it has right and left limits everywhere. The right and left limits of a function at $t$ are denoted by $f\left(t^{+}\right):=\lim _{s \searrow t} f(s)$ and $f\left(t^{-}\right):=\lim _{s} \nearrow_{t} f(s)$, respectively, provided they exist. In this notation, right continuity of $f(\cdot)$ in $t$, means that $f\left(t^{+}\right)=f(t)$.

For an interval $I \subseteq \mathbb{R}$, we denote by $\mathcal{L}^{1}\left(I, \mathbb{R}^{n} ; \mu\right)$ and $\mathcal{L}_{\text {loc }}^{1}\left(I, \mathbb{R}^{n} ; \mu\right)$ the space of integrable and locally integrable functions, respectively, from $I$ to $\mathbb{R}^{n}$ with respect to the measure $\mu$. If the measure is not specified then the integration is with respect to the Lebesgue measure. An absolutely continuous (AC) function $f:[a, b] \rightarrow \mathbb{R}^{n}$ is a function that can be written as $f(x)-f(a)=\int_{x}^{a} \dot{f}(t) d t$ for any $x \geq a$ for a function $\dot{f}(\cdot) \in \mathcal{L}^{1}\left([a, b], \mathbb{R}^{n}\right)$, which is considered as its derivative. The space of continuously differentiable functions is denoted by $\mathcal{C}^{1}\left(\mathbb{R}^{n}, \mathbb{R}^{m}\right)$, for $m, n \in \mathbb{N}$.

If $v: I \rightarrow \mathbb{R}^{n}$ is a function of bounded variation, then one can associate with it a Lebesgue-Stieltjes measure or the socalled differential measure $d v$ on $I$. Also, if $v(\cdot)$ is $r c b v$ on $[a, b]$, then we have the relation that $v(b)=v(a)+\int_{(a, b]} d v$.

The density of the measure $d v$ with respect to a positive Radon measure $\mu$ over an interval $I$ is defined as:

$$
\frac{d v}{d \mu}(t):=\lim _{\varepsilon \rightarrow 0} \frac{d v(I(t, \varepsilon))}{\mu(I(t, \varepsilon))},
$$

where $I(t, \varepsilon):=I \cap[t-\varepsilon, t+\varepsilon]$. Similarly, one can define the density of the Lebesgue measure $d t$ with respect to the Radon measure $\mu$. A Radon measure $\nu$ is absolutely continuous with respect to $\mu$ if for every measurable set $\mathcal{A}$, $\mu(\mathcal{A})=0$ implies that $\nu(\mathcal{A})=0$. Further, the measure $\nu$ is absolutely continuous with respect to $\mu$ if and only if the density function $\frac{d \nu}{d \mu}(\cdot)$ is well-defined (finite $\mu$ - almost everywhere) and is $d \mu$ integrable.

For a set $V \subset \mathbb{R}^{n}$, we will denote its interior by int $V$, and the boundary of this set is denoted by $\partial V$. If $V$ is convex, then $N_{V}(v)$ denotes the normal cone to $V$ at $v \in V$ and is defined as:

$$
N_{V}(v):=\left\{w \in \mathbb{R}^{n} \mid\langle w, x-v\rangle \leq 0 \forall x \in V\right\},
$$

where $\langle\cdot, \cdot\rangle$ denotes the usual inner product in $\mathbb{R}^{n}$.

\section{SOLUTION FrAMEWORK}

In this section, we will describe the dynamics of nonsmooth Lagrangian systems using differential inclusions and briefly talk about their solutions. The observer will then be designed using this formalism.

\section{A. Mathematical model}

We consider mechanical systems with $n$-degrees of freedom that are subjected to some unilateral constraints. The position variable $q \in \mathbb{R}^{n}$ is thus assumed to evolve in a set that admits the following form:

$$
\Phi:=\left\{q \in \mathbb{R}^{n} \mid h_{i}(q) \geq 0, i=1,2, \ldots, m\right\} .
$$

The geometry of the set $\Phi$ is determined by the functions $h_{i}(\cdot)$, and the only condition we will impose on the functions $h_{i}(\cdot)$ is that they are continuously differentiable so that $\nabla h_{i}(\cdot)$ is continuous for each $i$. This allows us to model a large number of closed domains which may even be nonconvex.

The convex polyhedral tangent cone $V(q)$ to the region $\Phi$ at a point $q$ is given by:

$$
V(q):=\left\{v \in \mathbb{R}^{n} \mid v^{\top} \nabla h_{i}(q) \geq 0, \forall i \in \mathcal{J}(q)\right\}
$$

where the set $\mathcal{J}(q)$ denotes the set of active constraints at $q$, i.e.,

$$
\mathcal{J}(q):=\left\{i \in\{1, \ldots, m\}: h_{i}(q) \leq 0\right\} .
$$

One can think of the set $V(q)$ as the set of admissible velocities that keep the position variable $q$ inside the set $\Phi$. The closed convex polyhedral cone $V^{\circ}(q)$, polar to $V(q)$ with respect to usual inner product on $\mathbb{R}^{n}$, is given by:

$$
V^{\circ}(q):=\left\{w \in \mathbb{R}^{n}: w^{\top} v \leq 0, \forall v \in V(q)\right\},
$$

that is, $V^{\circ}(q)$ is the outward normal cone to $\Phi$ at $q$, and is generated by the vectors $\nabla h_{i}(q), i \in \mathcal{J}(q)$. It is noted that $V(q)=\mathbb{R}^{n}$ and $V^{\circ}(q)=\{0\}$ if $q \in$ int $\Phi$.

We now formulate the dynamics of system (1) as a measure differential inclusion:

$$
\begin{aligned}
d q & =v d t \\
M(q) d v+F(t, q, v) d t & \in-N_{V(q)}\left(v_{e}\right) \subseteq V^{\circ}(q)
\end{aligned}
$$


where

$$
v_{e}(t):=\frac{v\left(t^{+}\right)+e v\left(t^{-}\right)}{1+e},
$$

and $e \in[0,1]$ is the coefficient of restitution. The initial condition is assumed to satisfy $q_{0}:=q(0) \in \Phi$, and $v_{0}:=$ $v(0) \in V\left(q_{0}\right)$. Since $V(q(t))$ is convex, the normal cone $N_{V(q(t))}$ at each $t$ is defined as in (3). To make connection to Lagrangian dynamical systems, in (7b), it is assumed that $F(t, q, v)=C(q, v) v+f(t, q, v)$ where $C(q, v) v$ denote the Coriolis and centrifugal torques.

The formulation for constrained mechanical systems, as in (7), was pioneered by J. J. Moreau [14], and the MDI (7) is called a second order sweeping process (because the constraint set for velocities appearing in (7b) depends on the state variable $q(\cdot)$ ). The motivation for working with the MDI is that we are seeking a solution to the evolution problem in the space of locally $r c b v$ functions to deal with possible collisions with the boundary of the admissible set. Functions which are locally $r c b v$ possess generalized derivatives that can be identified with Stieltjes measure and equation (7b) precisely describes the inclusion of the measure $d v$, associated with $v(\cdot)$, into a normal cone described by the constraint set $\Phi$. It is also noted that the post-impact velocity determined according to Moreau's collision rule (or Newton's impact law) is directly encoded in the MDI (7). Further details on inclusions of type (7) and comparisons with other modeling frameworks could be found in [5]. For our purpose, it is seen that the observer design given in Section IV is partially aided by this compact formulation.

\section{B. Existence of Solution}

The solution of MDI (7) is considered in the following sense:

Definition 1: A solution to the Cauchy problem (7) with initial data $\left(q_{0}, v_{0}\right) \in \Phi \times V\left(q_{0}\right)$, over an interval $I=$ $[0, T]$, is a pair $(q, v)$ such that $v(\cdot)$ is $r c b v$ on $I ; q(t)=$ $q_{0}+\int_{0}^{t} v(s) d s$; and furthermore, there exists a positive measure (represented by) $d \mu$ such that both $d t$ and $d v$ possess densities with respect to $d \mu$, denoted by $d t / d \mu$, and $d v / d \mu$ respectively, and satisfy

$M(q) \frac{d v}{d \mu}(t)+F(t, q, v) \frac{d t}{d \mu}(t) \in N_{V(q(t))}\left(v_{e}\right), d \mu$-a.e. on $I$.

The choice of the measure $d \mu$ is not unique since the righthand side of (7) is a cone. However, by Lebesgue-RadonNikodym theorem, the functions $d t / d \mu(\cdot) \in \mathcal{L}^{1}(I, R ; d \mu)$ and $d v / d \mu(\cdot) \in \mathcal{L}^{1}\left(I, \mathbb{R}^{n} ; d \mu\right)$ are uniquely determined for a given $d \mu$.

The problem of existence of solutions for evolution problems (1) has been studied for a long time. Earlier results on this problem dealt with the single constraint case $(m=1)$ and one may refer to [13, Chapter 3], [19] for results in this direction. The basic idea in these works is to introduce a time discretization scheme, either at position level [19] or velocity level [13] to construct a sequence of approximate solutions which is shown to converge as the sampling interval converges to zero. For several unilateral constraints $(m \geq 2)$, the existence and uniqueness has been proved in [2] under analytic assumptions on the data using the solution theory for differential equations and variational inequalities. The most relaxed conditions, under which the existence of solutions has been proved using discretization at velocity level, have appeared recently in [8], [18]. Based on the work of [18], the following regularity assumptions are required on the system data for the existence of solution, and are also needed for the observer design:

(H1) The function $F(\cdot, \cdot, \cdot)$ is continuous and is continuously differentiable $\left(\mathcal{C}^{1}\right)$ with respect to its second and third arguments.

(H2) The mapping $M(\cdot)$, from $\mathbb{R}^{n}$ to the set of symmetric positive definite matrices, belongs to class $\mathcal{C}^{1}$ and

$$
\underline{\lambda}_{M}|v|^{2} \leq v^{\top} M(q) v \leq \bar{\lambda}_{M}|v|^{2} \quad \forall(q, v) \in \Phi \times \mathbb{R}^{n} .
$$

(H3) For each $i \in\{1, \ldots, m\}$, the function $h_{i} \in$ $\mathcal{C}^{1}\left(\mathbb{R}^{n}, \mathbb{R}\right), \nabla h_{i}(q)$ is locally Lipschitz continuous and does not vanish in a neighborhood of $\left\{q \in \mathbb{R}^{n}: h_{i}(q)=0\right\}$.

(H4) The active constraints are functionally independent, i.e., $\left\{\nabla h_{i}(q)\right\}_{i \in \mathcal{J}(q)}$ is linearly independent for all $q \in \Phi$.

Without recalling the formal result on existence and assuming that a solution exists in the sense of Definition 1 under hypotheses $(\mathrm{H} 1)-(\mathrm{H} 4)$, we only collect the properties of the solutions to system (7) which provide more insight.

\section{Solution Characteristics}

1) The post-impact velocity: The condition, $q(t) \in \Phi$, for all $t$, allows for jumps in the velocity when $q$ hits the boundary of $\Phi$. At that instant, one must have $v\left(t^{-}\right) \in$ $-V(q(t))$, and $v\left(t^{+}\right) \in V(q(t))$. According to (7b), the jumps in $v(\cdot)$ satisfy:

$$
M(q(t))\left(v\left(t^{+}\right)-v\left(t^{-}\right)\right) \in-N_{V(q(t))}\left(v_{e}(t)\right),
$$

If there is only one active constraint, i.e., $\mathcal{J}(q(t))=\{i\}$, for some fixed $i \in\{1, \ldots, m\}$, then $V(q)=\mathbb{R}_{+} \nabla h_{i}(q(t))$, or equivalently, $N_{V(q(t))}(v(t))=\mathbb{R}_{-} \nabla h_{i}(q(t))$. These relations imply that

$$
v\left(t^{+}\right)=-e v\left(t^{-}\right)+(1+e) \operatorname{proj}_{M(q(t))}\left(V(q(t)), v\left(t^{-}\right)\right),
$$

where $\operatorname{proj}_{M(q)}(V(q))$ denotes the projection on $V(q)$ according to the kinetic metric at $q$, which is defined by the inner product $\langle v, w\rangle_{M(q)}=\langle v, M(q) w\rangle=\langle M(q) v, w\rangle$.

2) Regularity of state trajectories: The function $q$ is absolutely continuous, but not necessarily everywhere differentiable. The velocity $v(\cdot)$ is a locally $r c b v$ function, for which the left and right limits are defined everywhere. The acceleration is represented by the measure $d v$ and can be decomposed as a sum of two measures: an atomic measure $d \mu_{a}$ and Lebesgue-integrable function $\dot{v}(\cdot)$, i.e., $d v=d \mu_{a}+$ $\dot{v} d t$.

3) Countably many impacts: The set of impact times, at which $v(\cdot)$ is discontinuous, is countable. One may simply take $d \mu_{a}=\sum_{k \geq 0}\left[v\left(t_{k}^{+}\right)-v\left(t_{k}^{-}\right)\right] \delta_{t_{k}}$, where $\delta_{t_{k}}$ is the Dirac impulse at time $t_{k}$ and $\left\{t_{k}\right\}_{k \geq 0}$ is an ordered sequence of impact times. Thus, the formulation (7) does not exclude the 
Zeno phenomenon (with left accumulation point). However, if $e=1$, then there exists a constant $\rho>0$ such that $t_{k+1}-$ $t_{k}>\rho$, for each $k \geq 0$.

4) Non-uniqueness and Continuity of solutions: The solution of system (7) is unique if the system data is analytic [2], but in general, it may not be the case. Even under the analyticity assumption, the solutions may not vary continuously with respect to initial conditions under the hypotheses (H1) (H4). For this to hold, there is an additional condition on the set $\left\{\nabla h_{i}(q)\right\}_{i \in \mathcal{J}(q)}$ which states that for $e=0$, the active constraints must satisfy $\left\langle\nabla h_{i}(q), M^{-1}(q) \nabla h_{j}(q)\right\rangle \leq 0$, and for $e \in(0,1],\left\langle\nabla h_{i}(q), M^{-1}(q) \nabla h_{j}(q)\right\rangle=0$. The work of [9] assumes this condition because their design is based on closeness of solutions (in graphical sense) with respect to initial conditions. However, our observer design doesn't require this property and hence no such condition is imposed in our results.

\section{OBSERVER DESIGN}

We now address the problem of designing observers for the systems considered in Section III. It will be assumed that the position $q(\cdot)$ is the measured variable, and the objective is then to design an estimator for the velocity $v(\cdot)$ of the moving particle. In addition, we suppose that the following assumption holds:

Assumption 1: The velocity $v(\cdot)$ obtained as a solution to (7) stays bounded, that is,

$$
v(t) \in \mathcal{B}_{v}:=\left\{v \in \mathbb{R}^{n}:|v| \leq C_{v}\right\} \quad \forall t \geq 0,
$$

and furthermore, there exists a constant $C_{M}>0$ s.t.

$$
\|C(q, v)\| \leq C_{M}|v|, \quad \forall v \in \mathcal{B}_{v} .
$$

Before describing the observer dynamics, define the function $v \mapsto g(q, v)=C(q, v) v$ and let $\bar{f}(t, q, \cdot)$ and $\bar{g}(q, \cdot)$ denote the Lipschitz extensions ${ }^{1}$ of $f(t, q, \cdot)$ and $g(q, \cdot)$ from $\mathcal{B}_{v}$ with Lipschitz constants $C_{f}(t, q)$ and $C_{g}(q)$, respectively, and let $\bar{F}(t, q, \cdot)=\bar{f}(t, q, \cdot)+\bar{g}(q, \cdot)$. The observer we propose is:

$$
M(q) d z+\bar{F}(t, q, \hat{v}) d t-M(q) L \hat{v} d t \in-N_{V(q)}\left(\hat{v}_{e}\right)
$$

where

$$
\begin{aligned}
\hat{v}(t) & :=z(t)-L q(t), \\
\hat{v}_{e}(t) & :=\frac{\hat{v}\left(t^{+}\right)+e \hat{v}\left(t^{-}\right)}{1+e}
\end{aligned}
$$

and $q(\cdot)$ in (13a) and (13b) is an absolutely continuous function of time which is obtained from (7) as the measured output. The initial condition $\hat{v}_{0}:=\hat{v}(0) \in \mathcal{B}_{v}$. The design criteria for the output injection matrix $L$ will appear later in Section IV-B. The solution to system (13) is interpreted in the sense similar to Definition 1.

In the sequel, we will show that the proposed observer (13) has the following two properties:

\footnotetext{
${ }^{1}$ For a locally Lipschitz function $F: \mathcal{B} \rightarrow \mathbb{R}^{n}$, the function $\bar{F}: \mathbb{R}^{n} \rightarrow$ $\mathbb{R}^{n}$ is called the Lipschitz extension of $F(\cdot)$ from $\mathcal{B} \subset \mathbb{R}^{n}$ if $\bar{F}(\cdot)$ is globally Lipschitz over $\mathbb{R}^{n}$ and $\bar{F}(v)=F(v)$ for all $v \in \mathcal{B}$.
}

- Well-posedness: For each absolutely continuous function $q(\cdot)$, there exists a unique locally $r c b v$ function $\hat{v}(\cdot)$ that satisfies (13).

- Error convergence: The estimate $\hat{v}(\cdot)$ converges to $v(\cdot)$ asymptotically, i.e., $\lim _{t \rightarrow \infty}|v(t)-\hat{v}(t)|=0$.

In the following two subsections, it is proved that both these properties indeed hold. Before proceeding, note that the original system may not have unique solutions, but the observer has the property that it generates a unique trajectory corresponding to the each function $q(\cdot)$ observed as an output of system (7); see [6, Remark 3.3] for further explanation along these lines.

\section{A. Observer Well-posedness}

It is noted that the function $q(\cdot)$ is seen as an external "input" by the observer and hence $V(q(\cdot))$ is seen as a timeparameterized multi-valued function that does not depend on any of the internal states of the estimator. This makes the observer (13) a sweeping process of first order and the solutions for such systems are relatively easier to study. We have the following result:

Proposition 1: Assume that hypotheses (H1) - (H4) and Assumption 1 hold, then the system (13) is well-posed, that is, there exists a unique locally $r c b v$ solution $z:[0, \infty) \rightarrow \mathbb{R}$. Moreover, it holds that

$$
\hat{v}_{e}(t) \in V(q(t)) \quad \forall t \geq 0
$$

The basic idea in proving the existence of solutions of such systems is to construct a sequence of approximate solutions using the time-discretization scheme and show that the limit of that sequence is a solution to (13). These arguments are quite technical and the proof has been omitted due to space limitations. The relation (14), however, automatically holds for any solution of (13). We remark that, unlike (7), the uniqueness of solutions holds for system (13).

\section{B. Error Analysis}

The main result on convergence of error now follows:

Proposition 2: Assume that hypotheses (H1) - (H4) and Assumption 1 hold. If there exist a constant $\beta>0$ and a matrix $L$ such that, for all $(t, q) \in \mathbb{R}_{+} \times \Phi$,

$$
M(q) L+L^{\top} M(q) \leq-\left(2 C_{M} C_{v}+2 C_{f}(t, q)+2 C_{g}(q)+\beta\right) I_{n},
$$

where $I_{n} \in \mathbb{R}^{n \times n}$ denotes the identity matrix, then the velocity estimate $\hat{v}(\cdot)$ given by (13) converges to $v(\cdot)$ exponentially, that is, for some $c>0$,

$$
|v(t)-\hat{v}(t)| \leq c e^{-\beta t}|v(0)-\hat{v}(0)|
$$

Proof: Define the state estimation error as follows:

$$
\tilde{v}:=v-\hat{v}
$$

Then the dynamics for velocity estimation error are:

$$
\begin{aligned}
M(q) d \tilde{v}= & M(q) d v-M(q) d \hat{v} \\
= & M(q) d v-M(q) d z+M(q) L d q \\
= & M(q) L \tilde{v} d t-(F(t, q, v)-\bar{F}(t, q, \hat{v})) d t \\
& -(\eta-\hat{\eta})
\end{aligned}
$$


where

$$
\eta \in N_{V(q)}\left(v_{e}\right) \quad \text { and } \quad \hat{\eta} \in N_{V(q)}\left(\hat{v}_{e}\right)
$$

and $v_{e}, \hat{v}_{e}$ are defined as in (7c) and (13c), respectively. In what follows, we fix $d \mu=d t+d \mu_{a}+d \hat{\mu}_{a}$, where the atomic measure $d \mu_{a}$ (respectively $d \hat{\mu}_{a}$ ) is supported at the time instants where $v(\cdot)$ (respectively $\hat{v}(\cdot))$ is discontinuous. It is seen that $d t+d \mu_{a}$, and $d t+d \hat{\mu}_{a}$ are absolutely continuous with respect to $d \mu$ and hence the densities $\frac{d v}{d \mu}(\cdot)$ and $\frac{d \hat{v}}{d \mu}(\cdot)$ are well-defined.

Let $W:[0, \infty) \rightarrow \mathbb{R}_{+}$be defined as $W(t):=$ $\tilde{v}(t)^{\top} M(q(t)) \tilde{v}(t)$, then $W(\cdot)$ is locally $r c b v$ using the chain rule [15, Theorem 3], and its differential of the composition function is computed as follows:

$$
\begin{aligned}
\frac{d W}{d \mu}(t)= & \left(\tilde{v}\left(t^{+}\right)+\tilde{v}\left(t^{-}\right)\right)^{\top} M(q(t)) \frac{d \tilde{v}}{d \mu}(t) \\
& +\frac{\partial}{\partial q}\left(\tilde{v}\left(t^{+}\right)^{\top} M\left(q(t) \tilde{v}\left(t^{+}\right)\right) \frac{d q}{d \mu}(t) .\right.
\end{aligned}
$$

At time $t_{k}$, if there is a jump in $v(\cdot)$ or $\hat{v}(\cdot)$, then $\frac{d t}{d \mu}\left(t_{k}\right)=0$, which also implies that $\frac{d q}{d \mu}=\frac{d q}{d t} \cdot \frac{d t}{d \mu}=0$, and from (17c), we thus obtain:

$$
\begin{aligned}
\frac{d W}{d \mu}\left(t_{k}\right) & =\left(\tilde{v}^{+}+\tilde{v}^{-}\right)^{\top} M\left(q\left(t_{k}\right)\right) \frac{d \tilde{v}}{d \mu}\left(t_{k}\right) \\
& =\left(\tilde{v}^{+}+\tilde{v}^{-}\right)^{\top} M\left(q\left(t_{k}\right)\right)\left(\tilde{v}^{+}-\tilde{v}^{-}\right),
\end{aligned}
$$

where we used the notation $\tilde{v}^{+}=\tilde{v}\left(t_{k}^{+}\right), \tilde{v}^{-}=\tilde{v}\left(t_{k}^{-}\right)$. By definition, it follows that

$$
\begin{aligned}
& \eta \in-N_{V(q(t))}\left(v_{e}\right) \Longleftrightarrow\left\langle\eta, v_{e}-\hat{v}_{e}\right\rangle \geq 0 \\
& \hat{\eta} \in-N_{V(q(t))}\left(\hat{v}_{e}\right) \Longleftrightarrow\left\langle\hat{\eta}, v_{e}-\hat{v}_{e}\right\rangle \leq 0
\end{aligned}
$$

which in turn implies that

$$
\left\langle\eta-\hat{\eta}, v_{e}-\hat{v}_{e}\right\rangle \geq 0
$$

or equivalently,

$$
\left\langle\eta-\hat{\eta}, \frac{\tilde{v}^{+}+e \tilde{v}^{-}}{1+e}\right\rangle \geq 0 .
$$

Substituting $\eta-\hat{\eta}=-M\left(q\left(t_{k}\right)\right)\left(\tilde{v}^{+}-\tilde{v}^{-}\right)$, (23) is rewritten as

$$
\left\langle M\left(q\left(t_{k}\right)\right)\left(\tilde{v}^{+}-\tilde{v}^{-}\right), \frac{\tilde{v}^{+}+e \tilde{v}^{-}}{1+e}\right\rangle \leq 0 .
$$

In general, for $e \in[0,1], a, b \in \mathbb{R}^{n}$, and a symmetric positive definite matrix $M$, one can show that,

$$
\begin{aligned}
& \left\langle M(a-b), \frac{(a+e b)}{(1+e)}\right\rangle \leq 0 \\
\Longleftrightarrow & a^{\top} M a-e b^{\top} M b \leq(1-e) a^{\top} M b \\
\Longleftrightarrow & a^{\top} M a-e b^{\top} M b \leq 2(1-e) a^{\top} M b-a^{\top} M a+e b^{\top} M b \\
\Longleftrightarrow & (1+e)\left(a^{\top} M a-b^{\top} M b\right) \leq-(1-e)(a-b)^{\top} M(a-b) \\
\Longleftrightarrow & |a|_{M}^{2}-|b|_{M}^{2} \leq-\frac{(1-e)}{(1+e)}|a-b|_{M}^{2}
\end{aligned}
$$

where $|\cdot|_{M}$ denotes the norm with respect to the matrix $M$. Using the fact that $M\left(q\left(t_{k}\right)\right)$ is a symmetric positive definite matrix, (24) is equivalent to

$$
\begin{aligned}
\frac{d W}{d \mu}\left(t_{k}\right) & =\left\langle M\left(q\left(t_{k}\right)\right)\left(\tilde{v}^{+}-\tilde{v}^{-}\right), \tilde{v}^{+}+\tilde{v}^{-}\right\rangle \\
& =\left|\tilde{v}^{+}\right|_{M\left(q\left(t_{k}\right)\right)}^{2}-\left|\tilde{v}^{-}\right|_{M\left(q\left(t_{k}\right)\right)}^{2} \\
& \leq-\frac{(1-e)}{(1+e)}\left|\tilde{v}^{+}-\tilde{v}^{-}\right|_{M\left(q\left(t_{k}\right)\right)}^{2} \leq 0 .
\end{aligned}
$$

If $t \neq t_{k}$, then $\frac{d t}{d \mu}(t)=1$, and we let

$$
\frac{\partial}{\partial q}(\tilde{v}(t) M(q(t)) \tilde{v}(t)) \frac{d q}{d t}(t)=\tilde{v}(t) \dot{M}(q(t), v(t)) \tilde{v}(t)
$$

which results in

$$
\begin{aligned}
\frac{d W}{d \mu}(t) & =\frac{d W}{d t}(t)=\tilde{v}^{\top}\left(M(q) L+L^{\top} M(q)\right) \tilde{v}-\tilde{v}^{\top}(\eta-\hat{\eta}) \\
& -2 \tilde{v}^{\top}(F(t, q, v)-\bar{F}(t, q, \hat{v}))+\tilde{v}^{\top} \dot{M}(q(t), v(t)) \tilde{v}
\end{aligned}
$$

where $\tilde{v}$ and $q$ are evaluated at time $t$ and the argument has been suppressed for conciseness. In the above expression, $\tilde{v}^{\top}(\eta-\hat{\eta}) \geq 0$ because $v(t)=v_{e}(t)$ and $\hat{v}(t)=\hat{v}_{e}(t)$ are contained in $V(q(t))$ for all continuity points $t \geq 0$, due to which $\langle\eta, v-\hat{v}\rangle \geq 0$, and $\langle\hat{\eta}, v-\hat{v}\rangle \leq 0$. Adding and subtracting the term $2 \tilde{v}^{\top} C(q, v) \tilde{v}$ from the right-hand side, and using the fact that $\dot{M}(q, v)-2 C(q, v)$ is a skew symmetric operator, we obtain

$$
\begin{aligned}
\frac{d W}{d \mu}(t)=\tilde{v}^{\top} & \left(M(q) L+L^{\top} M(q)\right) \tilde{v} \\
& -2 \tilde{v}^{\top}(F(t, q, v)-\bar{F}(t, q, \hat{v})-C(q, v) \tilde{v})
\end{aligned}
$$

where we rewrite the norm of the last term as follows:

$$
\begin{aligned}
& |F(t, q, v)-\bar{F}(t, q, \hat{v})-C(q, v) \tilde{v}| \\
= & |g(q, v)-\bar{g}(q, \hat{v})-C(q, v) \tilde{v}+f(t, q, v)-\bar{f}(t, q, \hat{v})| \\
= & C_{g}(q)|\tilde{v}|+C_{M} C_{v}|\tilde{v}|+C_{f}(t, q)|\tilde{v}|
\end{aligned}
$$

It follows under condition (15) that

$$
\frac{d W}{d \mu}(t) \leq-\beta W(t), \quad t \neq t_{k} .
$$

Since we fixed $d \mu=d t+d \mu_{a}+d \hat{\mu}_{a}$, and $W(\cdot)$ is nonincreasing at the atoms of $d \mu_{a}$ and $d \hat{\mu}_{a}$ because of (25), and decreasing exponentially with respect to Lebesgue measure due to (26). This results in

$$
W(t) \leq e^{-\beta t} W(0) \Rightarrow|\tilde{v}(t)| \leq \sqrt{\frac{\bar{\lambda}_{M}}{\underline{\lambda}_{M}}} e^{-\beta t}|\tilde{v}(0)|
$$

showing that the error $\tilde{v}(\cdot)$ decays exponentially.

Example 1: We now apply our result to a bouncing ball which is a one degree of freedom system with a single constraint. We let $m(q)=1, h(q)=q \geq 0$, so that $V(q)=\mathbb{R}$ if $q>0$, and $V(q)=\mathbb{R}_{+}$if $q \leq 0$. This leads to

$$
N_{V(q)}(v)= \begin{cases}\{0\}, & \text { if } q>0, \text { or } v>0, \\ \mathbb{R}_{-}, & \text {if } q \leq 0, \text { and } v \leq 0 .\end{cases}
$$




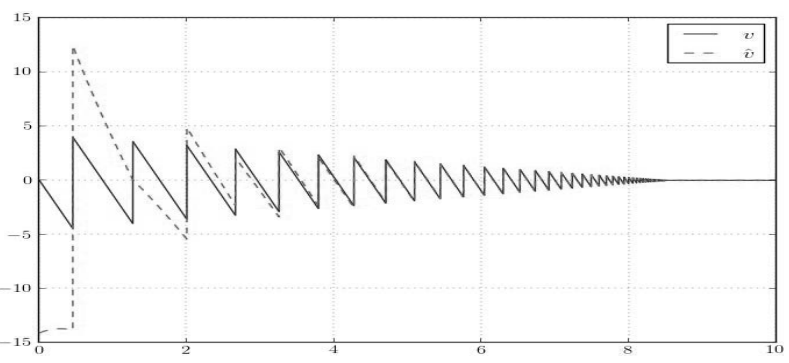

Fig. 1. Simulation of velocity estimator for bouncing ball with $e=0.9$.

In the form of (7), the dynamics could be described by $d q=$ $v$ and

$$
d v+g d t \in-N_{V(q)}(v) .
$$

If there is a discontinuity in $v(\cdot)$ at $t_{k}$, then $v\left(t_{k}^{-}\right)<0$, and the impact occurs when $q=0$, and at that instant

$$
v\left(t_{k}^{+}\right)-v\left(t_{k}^{-}\right) \in-N_{\mathbb{R}_{+}}\left(\frac{v\left(t_{k}^{+}\right)+e v\left(t_{k}^{-}\right)}{1+e}\right) .
$$

This is an implicit relation for $v\left(t_{k}^{+}\right)$, for which the only solution is $v\left(t_{k}^{+}\right)=-e v\left(t_{k}^{-}\right)$. The estimated velocity for this system is given by $\hat{v}(t)=z(t)-l q(t)$, where $z(\cdot)$ is obtained from

$$
d z+g d t-l z d t+l^{2} q d t \in-N_{V(q)}\left(\hat{v}_{e}\right)
$$

with $l<0$. The proposed observer has been simulated in SICONOS [1] and the simulation results are given in Figure 1. It is seen that the estimated velocity respects the same constraints as imposed on the actual velocity of the system. However, not every impact of the ball with the ground causes a discontinuity in $\hat{v}(\cdot)$.

\section{CONCLUSiOns}

The problem of designing asymptotically convergent state estimators for nonsmooth mechanical systems was considered in this paper. The formalism of differential inclusions was used for both the system and observer dynamics. For the later, we proved the existence and uniqueness of solutions. The error analysis (for the convergence of velocity estimate) was based on generalizing the Lyapunov techniques to functions of locally bounded variation.

\section{ACKNOWLEDGEMENTS}

The first author would like to thank Prof. Manuel D. P. Monteiro-Marques for useful discussions related to the proof of Proposition 1.

\section{REFERENCES}

[1] V. Acary and F. Pérignon. An introduction to SICONOS. Technical Report RR - 0340, INRIA, Rhône-Alpes, 2007. Available online: http://hal.inria.fr/inria-00162911. Project website: http://siconos.gforge.inria.fr.

[2] P. Ballard. The dynamics of discrete mechanical systems with perfect unilateral constraints. Arch. Rational Mech. Anal., 154:199 - 274, 2000.

[3] H. Berghuis and H. Nijmeijer. A passivity approach to controllerobserver design for robots. IEEE Trans. Robotics and Automation, 9(6):740 - 754, 1993.

[4] G. Besancon. Global output feedback tracking control for a class of Lagrangian systems. Automatica, 38:1915 - 1921, 2002.
[5] B. Brogliato. Nonsmooth Mechanics. Springer-Verlag London, 2nd edition, 1999.

[6] B. Brogliato and W.P.M.H. Heemels. Observer design for Lur'e systems with multivalued mappings: A passivity approach. IEEE Trans. Automatic Control, 54(8):1996 - 2001, 2009.

[7] C. Castaing and M. D. P. Monteiro Marques. BV periodic solutions of an evolution problem associated with continuous moving convex sets. Set-Valued Analysis, 3:381 - 399, 1995.

[8] R. Dzonou, M. D. P. Monteiro Marques and L. Paoli. A convergence result for a vibro-impact problem with a general inertia operator Nonlinear Dynamics, 58: 361 - 384, 2009.

[9] F. Forni, A. Teel, and L. Zaccarian. Follow the bouncing ball: Global results on tracking and state estimation with impacts. IEEE Trans. Automatic Control, 58(6): 1470 - 1485, 2013.

[10] W. P. M. H. Heemels, M. K. Camlibel, J. M. Schumacher, and B. Brogliato. Observer-based control of linear complementarity systems. Int. J. Robust \& Nonlinear Control, 21(10):1193 - 1218, 2011.

[11] M. Kunze and M. D. P. Monteiro Marques. An introduction to Moreau's sweeping process. In B. Brogliato, editor, Impacts in Mechanical Systems: Analysis and Modelling, volume 551 of Lecture Notes in Physics, pages $1-60$. Springer-Verlag, Berlin, 2000.

[12] L. Menini and A. Tornambé. Velocity observers for non-linear mechanical systems subject to non-smooth impacts. Automatica, $38: 2169-2175,2002$

[13] M. D. P. Monteiro Marques. Differential Inclusions in Nonsmooth Mechanical Problems: Shocks and Dry Friction, volume 9 of Progress in Nonlinear Differential Equations and their Applications. Birkhäuser, 1993.

[14] J. J. Moreau. Unilateral contact and dry friction in finite freedom dynamics. In J. J. Moreau and P. D. Panagiotopoulos, editors, Nonsmooth Mechanics and Applications, volume 302 of CISM Courses and Lectures, International Centre for Mechanical Sciences, pages 1 - 82. Springer, Berlin, 1988.

[15] J. J. Moreau and M. Valadier. A chain rule involving vector functions of bounded variation. J. Functional Analysis, 74(2):333 - 345, 1987.

[16] S. Nicosia and P. Tomei. Robot control by using only joint position measurements. IEEE Trans. Automatic Control, 35(9):1058 - 1061, 1990.

[17] J. A. Osorio and J. A. Moreno. Dissipative design of observers for multivalued nonlinear systems. In Proc. 45th IEEE Conf. Decision \& Control, pages 5400 - 5405, San Diego (CA), USA, December 2006.

[18] L. Paoli. A proximal-like method for a class of second order measuredifferential inclusions describing vibro-impact problems. J. Differential Equations, 250:476 - 514, 2011.

[19] L. Paoli and M. Schatzman. A numerical scheme for impact problems I and II. SIAM J. Numer. Anal., 40(2):702 - 733, 734 - 768, 2002.

[20] H. Shim, Y. Son, and J. Seo. Semi-global observer for multi-output nonlinear systems. Sys. \& Control Letters, 42(3): 233 - 244, 2001.

[21] H. Shim and A. Tanwani. Hybrid-type observer design based on a sufficient condition for observability in switched nonlinear systems. Int. J. Robust \& Nonlinear Control: Special Issue on High Gain Observers and Nonlinear Output Feedback Control, 2012. DOI: 10.1002/rnc.2901; to appear.

[22] A. Tanwani. Invertibility and Observability of Switched Systems with Inputs and Outputs. $\mathrm{PhD}$ thesis, University of Illinois at Urbana-Champaign, IL, USA, 2011. Available online: http://hdl. handle.net/2142/29755.

[23] A. Tanwani, B. Brogliato, and C. Prieur. Stability and observer design for a class of nonsmooth dynamical systems with nonconvex state constraints. Research Report RR - 8248, INRIA, Rhône-Alpes, 2013. Available online: http://hal.inria.fr/hal-00790618.

[24] A. Tanwani, H. Shim, and D. Liberzon. Observability for switched linear systems: characterization and observer design. IEEE Trans. Automatic Control, 58(4): 891 - 904, 2013.

[25] A. Tanwani and S. Trenn. Observability of switched differentialalgebraic equations for general switching signals. In Proc. 51st IEEE Conf. Decision \& Control, pages 2648 - 2653, 2012.

[26] A. Tanwani and S. Trenn. An observer for switched differentialalgebraic equations based on geometric characterization of observability. In Proc. 52nd IEEE Conf. Decision \& Control, 2013.

[27] B. Xian, M. S. de Queiroz, D. M. Dawson, and M. L. McIntyre. A discontinuous output feedback controller and velocity observer for nonlinear mechanical systems. Automatica, 40:695 - 700, 2004. 